B. Kovalerchuk, W. Sumner, M. Curtiss, M. Kovalerchuk, and R. Chase In: Algorithms and technologies for multispectral, hyperspectral and ultraspectral imagery IX. Vol. 5425, International SPIE military and aerospace symposium, AEROSENSE, Orlando, FL, April 12-15, 2004, pp. 508-519•

\title{
MATCHING IMAGE FEATURE STRUCTURES USING SHOULDER ANALYSIS METHOD
}

\author{
Boris Kovalerchuk*, William Sumner, Mark Curtiss, Michael Kovalerchuk, Richard Chase \\ Dept. of Computer Science, Central Washington University, Ellensburg, WA, USA 98926-7520
}

\begin{abstract}
The problems of imagery registration, conflation, fusion and search require sophisticated and robust methods. An algebraic approach is a promising new option for developing such methods. It is based on algebraic analysis of features represented as polylines. The problem of choosing points when attempting to prepare a linear feature for comparison with other linear features is a significant challenge when orientation and scale is unknown. Previously we developed an invariant method known as Binary Structural Division (BSD). It is shown to be effective in comparing feature structure for specific cases. In cases where a bias of structure variability exists however, this method performs less well. A new method of Shoulder Analysis (SA) has been found which enhances point selection, and improves the BSD method. This paper describes the use of shoulder values, which compares the actual distance traveled along a feature to the linear distance from the start to finish of the segment. We show that shoulder values can be utilized within the BSD method, and lead to improved point selection in many cases. This improvement allows images of unknown scale and orientation to be correlated more effectively.
\end{abstract}

Keywords: data fusion, imagery conflation, algebraic invariants, geospatial feature, polyline match, measure of correctness, structural similarity, structural interpolation

\section{INTRODUCTION}

The registration and conflation method based on algebraic invariants uses polylines constructed from extracted features to compare and combine images ${ }^{19}$. Polyline comparisons may be done in several ways. The simplest way is to simply look at them. Consider the two satellite images of a lake in Kyrgyzstan in Fig. 1.

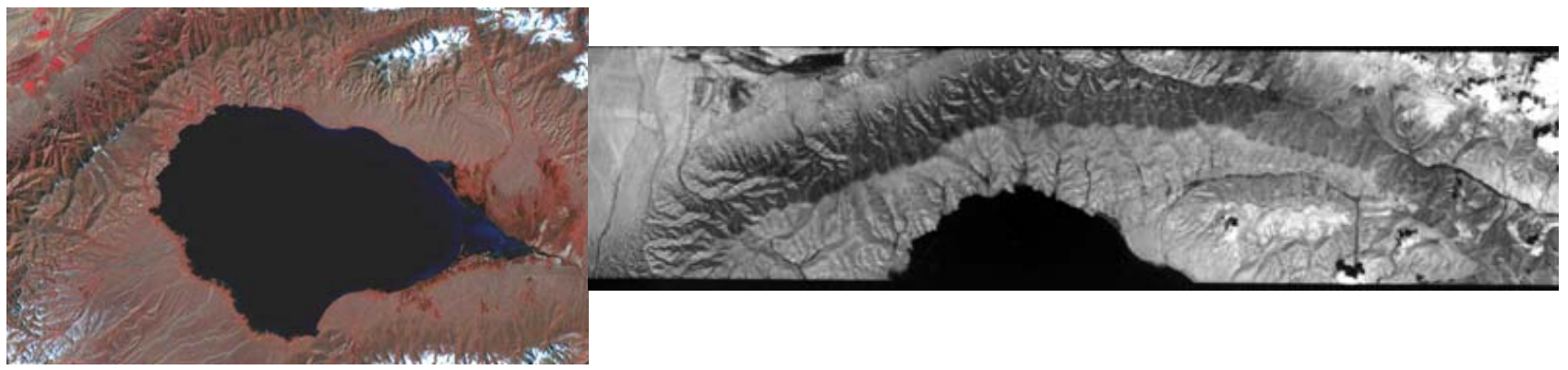

Fig. 1. Two images of the lake Sonkyl in Kyrgyzstan.

Feature extraction programs can be used to construct numerous polylines, with the most obvious one being the shoreline of the lake. The results are shown in Fig. 2.

\footnotetext{
*borisk@cwu.edu; phone 1509 963-1438; fax 1509 963-1449; www.cwu.edu
} 
While the overall structure of the extracted shorelines is apparent, the polylines differ in detail for a variety of reasons. Robust ways of comparing these polylines are necessary to determine image transformations and to assess the quality of the result.

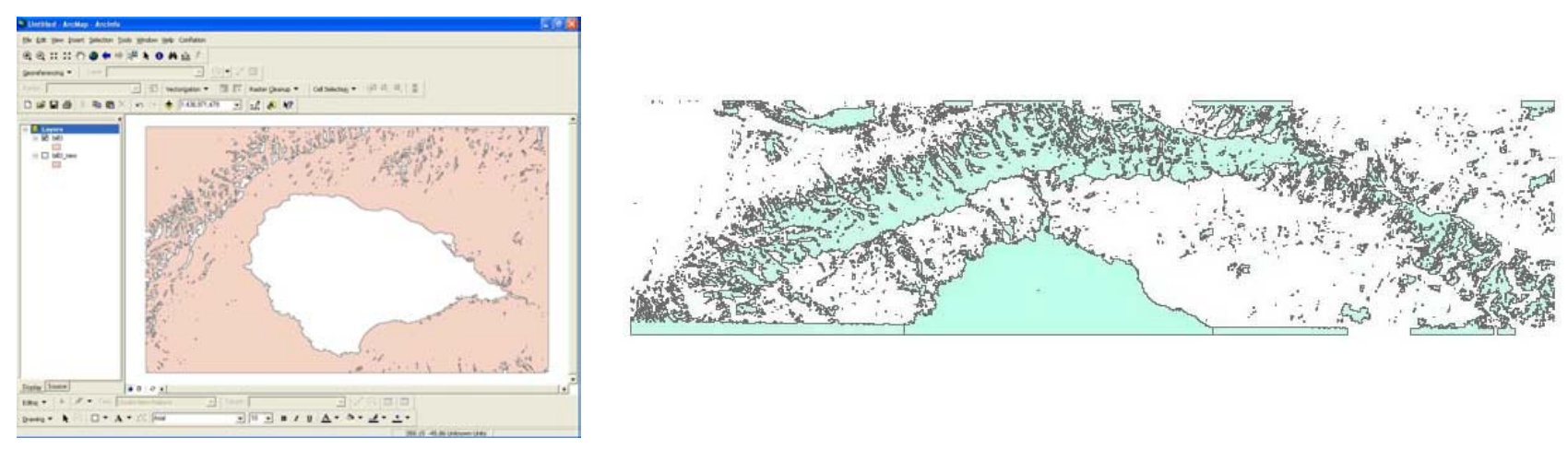

Fig. 2. Extracted features from the photographs of Sonkyl.
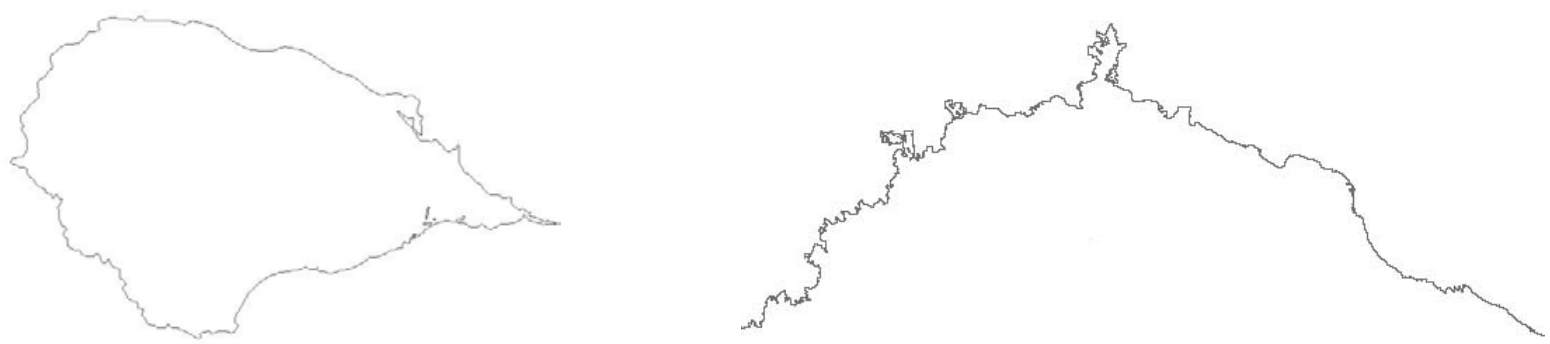

Fig. 3. Lake shores extracted from the photographs of Sonkyl.

The angles between segments and individual segment lengths are two algebraic characteristics of polylines that are used. For smooth features extracted from images with comparable scales and resolutions, either comparison works well. When there are marked differences in image scale and resolution, the choice of angles or lengths becomes more important. This paper examines characteristics of extracted polylines and how they may be interpolated and compared. For many cases, including the one illustrated here, comparisons based on segment lengths are shown to be superior.

\section{POLYLINE INTERPOLATIONS}

One common problem with polylines is discontinuity that results from image resolution, differences in image acquisition, and artifacts of feature extraction algorithms. Extracted features can be modified in two ways to give a common resolution complexity to facilitate comparisons.

Consider the case of a curvilinear feature that is segmented as a result of something obscuring it, such as a road shaded by a tree. By connecting segments that are "close enough" and have "small" deviation angles, a composite feature can be formed. The maximum separation distance and the maximum deviation angle parameters permitted are clearly critical to feature creation and to one's confidence in the result.

Another type of feature modification is necessary to simplify curvilinear features with one or more relatively narrow lobes for comparison to one with no narrow lobes. For example, a state road map will typically depict a coastline with very little structure. A high resolution aerial photograph, on the other hand, will show the same coastline with lots of structure, showing that it goes inland for miles along river channels and juts out around spits of land. The higher resolution feature can be simplified by removing these lobes if they are "sufficiently narrow." Critical parameters here are the unit size of feature sampling and maximum jumping distance permitted.

With images prepared with this preprocessing, it is possible to register images that appear at first to have few if any features in common and are of unknown scale and orientation. 
Measures of spatial similarity of polylines also need to be developed. Here, the focus is on spatial similarity characteristics while the similarity of non-spatial feature attributes can be matched after a spatial match is confirmed. If two images are matched using only a few reference points, the similarity of other points also needs to be assessed.

The issue of variability of points that form a polyline also needs to be addressed. Different feature extraction algorithms and imagery analysts can assign points differently on the same physical feature. This can affect finding coreference candidate features. The technique described below addresses this problem in a computationally efficient way.

Consider the polyline in Fig. 4 and the successive approximations defined by taking points defined by the mid point of lengths along the polyline as indicated. The method is called Binary Sequential Division (BSD) method $^{8}$ computed by finding a curve middle point along the curve, then repeated for each half, halves of halves and so on.

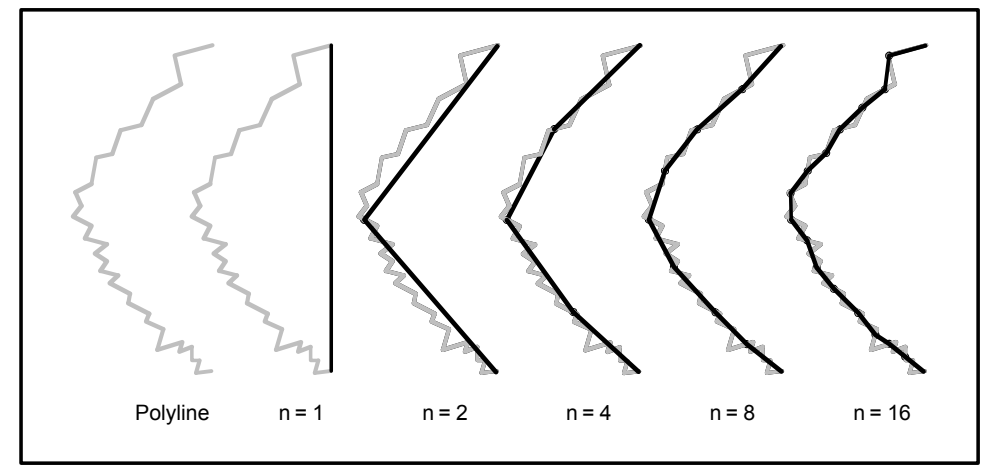

Fig. 4. Structural interpolations of a polyline. Our experiments show that 8 binary sequential divisions with $256=2^{8}$ linear segments is typically sufficient for interpolation.

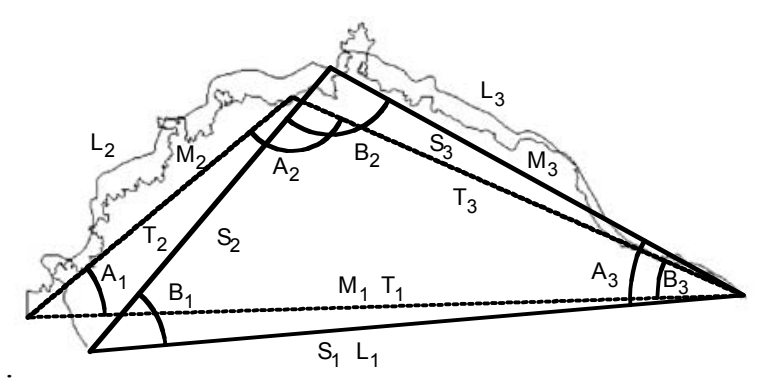

Fig. 5. Sections of the extracted shorelines with the first level BSD interpolation with $\mathrm{k}=1$ and $\mathrm{n}=2$.

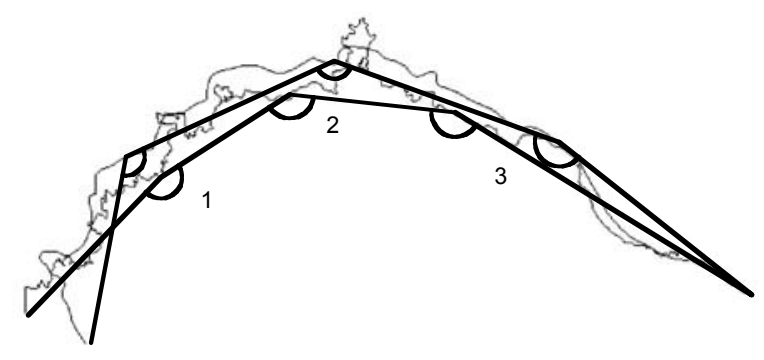

Fig. 6. Fragment of BSD level 2 for the two polylines.

$G(n)$ is used to denote the $n$-th interpolation of a polyline. As mentioned above $n=2^{k}$, and $k$ is called the BSD level. The first four steps of the conflation process are:

Step 1. For raster images extract several linear features as sets of points (pixels), S. For vector images skip this step.

Step 2. Vectorize extracted linear features. For vector images skip this step.

Step 3. For both raster and vector images analyze the complexity and connectivity of vectorized linear features. If features are too simple (contain few points and are small relative to the image size) combine several features in a superfeature. If features are too complex, simplify features by applying a gap analysis algorithm. In the ideal situation we also should be able to separate feature extraction algorithm artifacts from real features. In the example here, the algorithm introduced artifacts, by capturing vegetation as a part of the shoreline in several places.

Step 4. Interpolate each superfeature as a specially designed polyline using the BSD method.

Fig. 5 depicts level 1 BSD interpolation with $k=1$ and $n=2$ for the vectorized features shown in Fig. 3 . The middle points of each feature are as shown, computed along each line. Significant fluctuations have been lost in the lower 
resolution image. Feature $M$ as interpolated has angles $A_{1}, A_{2}$, and $A_{3}$. Feature $L$ as interpolated has angles $B_{1}, B_{2}$, and $\mathrm{B}_{3}$

\section{GENERATION OF MATRICES}

Matrices are constructed from the angle relationships and from the length relationships of the polylines by using two algorithms, denoted as the Angle Algorithm (AA) and the Shoulder Algorithm (SA). Matrix computation form steps 5 and 6 of the conflation algorithm:

Step 5. Compute a matrix $\mathrm{Q}$ of the relation between all angles on the polyline by using AA Algorithm.

Step 6. Compute a matrix $\mathrm{P}$ of the relation between all lengths of intervals on the polyline by using SA algorithm.

Values of 0 and 1 are used to indicate $\geq$ and $<$ respectively. For this example, the angular relations for the two polylines are presented in Table 1 and the length (or shoulder) relations are in Table 2.

\begin{tabular}{|c|c|c|c|}
\hline & $\mathrm{A} 1$ & $\mathrm{~A} 2$ & $\mathrm{~A} 3$ \\
\hline $\mathrm{A} 1$ & $\mathrm{~A} 1 \geq \mathrm{A} 1$ & $\mathrm{~A} 1<\mathrm{A} 2$ & $\mathrm{~A} 1 \geq \mathrm{A} 3$ \\
\hline $\mathrm{A} 2$ & $\mathrm{~A} 2 \geq \mathrm{A} 1$ & $\mathrm{~A} 2 \geq \mathrm{A} 2$ & $\mathrm{~A} 2 \geq \mathrm{A} 3$ \\
\hline $\mathrm{A} 3$ & $\mathrm{~A} 3<\mathrm{A} 1$ & $\mathrm{~A} 3<\mathrm{A} 2$ & $\mathrm{~A} 3 \geq \mathrm{A} 3$ \\
\hline
\end{tabular}

Table 1: Angular relations $A_{i} \geq A_{i}$ and $B_{i} \geq B_{i}$

Matrix of angular relations in feature in image A

\begin{tabular}{|c|c|c|c|}
\hline & $\mathrm{B} 1$ & $\mathrm{~B} 2$ & $\mathrm{~B} 3$ \\
\hline $\mathrm{B} 1$ & $\mathrm{~B} 1 \geq \mathrm{B} 1$ & $\mathrm{~B} 1<\mathrm{B} 2$ & $\mathrm{~B} 1 \geq \mathrm{B} 3$ \\
\hline $\mathrm{B} 2$ & $\mathrm{~B} 2 \geq \mathrm{B} 1$ & $\mathrm{~B} 2 \geq \mathrm{B} 2$ & $\mathrm{~B} 2 \geq \mathrm{B} 3$ \\
\hline $\mathrm{B} 3$ & $\mathrm{~B} 3<\mathrm{B} 1$ & $\mathrm{~B} 3<\mathrm{B} 2$ & $\mathrm{~B} 3 \geq \mathrm{B} 3$ \\
\hline
\end{tabular}

\begin{tabular}{|l|l|l|l|}
\hline & $\mathrm{B} 1$ & $\mathrm{~B} 2$ & $\mathrm{~B} 3$ \\
\hline $\mathrm{B} 1$ & 1 & 0 & 1 \\
\hline $\mathrm{B} 2$ & 1 & 1 & 1 \\
\hline $\mathrm{B} 3$ & 0 & 0 & 1 \\
\hline
\end{tabular}

\begin{tabular}{|l|l|l|l|}
\hline & A1 & A2 & A3 \\
\hline A1 & 1 & 0 & 1 \\
\hline A2 & 1 & 1 & 1 \\
\hline A3 & 0 & 0 & 1 \\
\hline
\end{tabular}

Matrix of angular relations in feature in image B

Table 2: Length (shoulder) relations $S_{i} \geq S_{i}$ and $T_{i} \geq T_{i}$

\begin{tabular}{|c|c|c|c|}
\hline & $\mathrm{S} 1$ & $\mathrm{~S} 2$ & $\mathrm{~S} 3$ \\
\hline $\mathrm{S} 1$ & $\mathrm{~S} 1 \geq \mathrm{S} 1$ & $\mathrm{~S} 1 \geq \mathrm{S} 2$ & $\mathrm{~S} 1 \geq \mathrm{S} 3$ \\
\hline $\mathrm{S} 2$ & $\mathrm{~S} 2<\mathrm{S} 1$ & $\mathrm{~S} 2 \geq \mathrm{S} 2$ & $\mathrm{~S} 2<\mathrm{S} 3$ \\
\hline $\mathrm{S} 3$ & $\mathrm{~S} 3<\mathrm{S} 1$ & $\mathrm{~S} 3 \geq \mathrm{S} 2$ & $\mathrm{~S} 3 \geq \mathrm{S} 3$ \\
\hline
\end{tabular}

Matrix of length relations in feature in image A

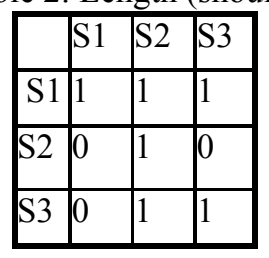

Table 3: Part of the BSD level 2 for the same features

\begin{tabular}{|c|c|c|c|}
\hline & $\mathrm{T} 1$ & $\mathrm{~T} 2$ & $\mathrm{~T} 3$ \\
\hline $\mathrm{T} 1$ & $\mathrm{~T} 1 \geq \mathrm{T} 1$ & $\mathrm{~T} 1 \geq \mathrm{T} 2$ & $\mathrm{~T} 1 \geq \mathrm{T} 3$ \\
\hline $\mathrm{T} 2$ & $\mathrm{~T} 2<\mathrm{T} 1$ & $\mathrm{~T} 2 \geq \mathrm{T} 2$ & $\mathrm{~T} 2<\mathrm{T} 3$ \\
\hline $\mathrm{T} 3$ & $\mathrm{~T} 3<\mathrm{T} 1$ & $\mathrm{~T} 3 \geq \mathrm{T} 2$ & $\mathrm{~T} 3 \geq \mathrm{T} 3$ \\
\hline
\end{tabular}

Image A: angles

$1: 2.206752$

2: 2.389911

3: 2.797306

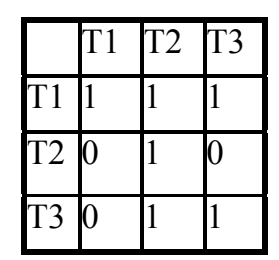

Matrix of length relations in feature in image B

\begin{tabular}{|l|l|l|l|}
\hline & 1 & 2 & 3 \\
\hline 1 & 1 & $\mathbf{1}$ & $\mathbf{1}$ \\
\hline 2 & & 1 & 0 \\
\hline 3 & & & 1 \\
\hline
\end{tabular}

Image B: angles

1: 2.906888

2: 2.467343

3: 2.702809

Matrices for features from images A and B for angles marked 1,2, and 3. Bold numbers indicate differences between angular relations in two features.

The tables above only show matrixes for BSD level 1. In the general case for BSD level $k$ there are $2^{k}-1=n-1$ segments and each matrix generated has size $(n-1) \times(n-1)$. Fig. 6 and Table 3 show a part of the BSD level 2 for the same features.

Another way to compare interpolated polylines is to construct a matrix of shoulder/length ratios $\mathrm{S} / \mathrm{L}$, where the length $\mathrm{L}$ is computed as a distance between the shoulder end points along the polyline. We use two relations: $\mathrm{S}_{\mathrm{i}} / \mathrm{L}_{\mathrm{i}} \geq \mathrm{S}_{\mathrm{j}} / \mathrm{L}_{\mathrm{j}}$ and $\left|\mathrm{S}_{\mathrm{i}} / \mathrm{L}_{\mathrm{i}}-\mathrm{S}_{\mathrm{j}} / \mathrm{L}_{\mathrm{j}}\right|<\varepsilon$. In the matrix for relation $\mathrm{S}_{\mathrm{i}} / \mathrm{L}_{\mathrm{i}} \geq \mathrm{S}_{\mathrm{j}} / \mathrm{L}_{\mathrm{j}}$ all diagonal cells $(i=j)$ are equal to 1 because $\mathrm{S}_{\mathrm{i}} / \mathrm{L}_{\mathrm{i}} \geq \mathrm{S}_{\mathrm{i}} / \mathrm{L}_{\mathrm{i}}$ is always true. Also $\mathrm{S}_{1} / \mathrm{L}_{1}=1$, since by definition for the base line $\mathrm{S}_{1}=\mathrm{L}_{1}$. 
Next, $L_{2}=L_{3}$ by the middle point design, thus $S_{3} / L_{3} \geq S_{2} / L_{2}$ is true if and only if $S_{3} \geq S_{2}$. We also know by definition that $1 \geq S_{i} / L_{i}$, because $S_{i}$ as a straight line is shorter or equal to the curve $L_{i}$ between the same points. This analysis shows that relations for ratios $\mathrm{S} / \mathrm{L}$ are the same as for $\mathrm{S}$. But ratios can provide more relations, for instance we may discover that $2 * S_{2}<S_{3}$, i.e., $S_{2}$ is twice smaller than $S_{1}$.

\section{TESTING DATA CLOSENESS}

Once the matrices have been generated, measures of closeness may be calculated for each polyline in one image with each polyline in the other. The AA and SA algorithms make these comparisons. These comparisons form steps 7 and 8 of the Conflation Method: Step 7. Testing closeness for angles; Step 8. Testing closeness for lengths.

In the example, Tables 1 and 2 show that matrixes for two features are identical in both images. This means that we have the highest closeness of given features on BSD level 1. However Table 3 reveals that matrixes for BSD level 2 are different and there is no match on this level. If there are successful matches, higher BSD levels are explored until the match fails. A match level tree that shows the deepest match level reached for each section on each BSD level can be used to make a final judgment about feature match.

The set of relations computed by the AA and SA algorithms in practice also include thresholds to make the methods more robust to noise and multi-resolution mismatch. This is illustrated in Table 4.

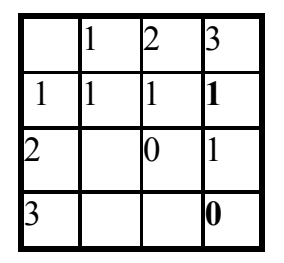

Table 4: Angle comparisons using thresholds

Level 2 BSD angle comparison matrix: image A (columns) and image B (rows) with threshold $=0.209440$. Note, 1 indicates the difference in the column and row values is greater than the threshold., $\left|\mathrm{L}_{\mathrm{i}}-\mathrm{L}_{\mathrm{j}}\right|<\varepsilon$. This matrix indicates that Level 2 BSD match fails. There is only one value in the threshold limits.

\section{MATCH FORECASTING AND EVALUATION}

\subsection{Prediction outline}

The goal of step 9 is to identify the deepest level of structural match for two features. The brute force version of this step is to repeat steps 7 and 8 for all deeper BSD level $k+1, k+2$ and so on. The more efficient version of step 9 described below first forecasts feature match on the next BSD level and computes the next BSD level only if the forecast is successful. Otherwise the forecasting algorithm explores potential match for halves of the feature (shoulders). If it fails too then the features are cut down as described in steps 10 and 11. Step 9 contains the following substeps:

9.1. If Steps 7 and 8 are successful on BSD level $k$, forecast potential match in the next BSD level $k+1$ using forecasting algorithm FA (see algorithm described below).

9.2. If match is forecasted by FA algorithm for BSD level $k+1$, repeat step 9 for BSD level $k+1$ until mismatch is forecasted.

If mismatch is forecasted by FA algorithm for BSD level $k+1$, use FA algorithm for match forecasting for respective halves of the features (right and left shoulders).

9.3. Construct a match level tree that shows the deepest match level reached for each section on each BSD level.

9.4. Evaluate the tree to make a final judgment about feature match.

Fig. 7 shows the final conflation result for the example discussed.

\subsection{Match prediction algorithm using SA algorithm}

Algorithm AA for BSD level $k=0$ provides us the lengths of three curves $\mathrm{L}_{1}, \mathrm{~L}_{2}$ and $\mathrm{L}_{3}$ (see Fig. 5), where $\mathrm{L}_{1}$ is the base line connecting two ends of the feature curve, and $\mathrm{L}_{2}=\mathrm{L}_{3}$ by the definition of the line middle point used to built them. Similarly SA algorithm for $k=0$ produces three straight lines (shoulders) $\mathrm{S}_{1}, \mathrm{~S}_{2}$ and $\mathrm{S}_{3}$ connecting two ends and the 
middle point of the feature curve (see Fig. 5). Similarly, for every other $k$, AA and SA algorithms provide a set of $\mathrm{L}_{\mathrm{i}}$ for each polyline segment along the curve and a set of shoulders $S_{i}$ between those segments (see an example in Fig. 6).

Next we compute all $\mathrm{S}_{\mathrm{i}} / \mathrm{L}_{\mathrm{i}}$ for BSD level $k=0$ for image $\mathrm{A}$ and similar ratios for image $\mathrm{B}$, denoted as $\mathrm{T}_{\mathrm{i}} / \mathrm{M}_{\mathrm{i}}$ in Fig. 5 . Say these ratios are 1, 0.5 and 0.8 for image $\mathrm{A}$ and $1,0.3$ and 0.83 for image $\mathrm{B}$.

After that we search for a pair of shoulders $<\mathrm{S}_{\mathrm{i}} / \mathrm{L}_{\mathrm{i}}, \mathrm{T}_{\mathrm{i}} / \mathrm{M}_{\mathrm{i}}>$ such that $\left|\mathrm{S}_{\mathrm{i}} / \mathrm{L}_{\mathrm{i}}-\mathrm{T}_{\mathrm{i}} / \mathrm{M}_{\mathrm{i}}\right|>\varepsilon$ in the two images. If shoulders are found, this is declared a mismatch forecast. If there are no such shoulders, the FA algorithm forecasts a potential match on the next level and the Conflation Method proceeds to compute BSD level $k+1$.

In the example above, $\left|\mathrm{S}_{2} / \mathrm{L}_{2}-\mathrm{T}_{2} / \mathrm{M}_{2}\right|=|0.5-0.3|>\varepsilon$, if $\varepsilon=0.1$. Thus means that the CM method will not compute BSD level $k+1=2$ for the whole feature but will compute BSD for the third section of the curve with $\left|\mathrm{S}_{3} / \mathrm{L}_{3}-\mathrm{T}_{3} / \mathrm{M}_{3}\right|=\mid 0.8$ $0.83 \mid<0.1$.

The motivation for this algorithm is the following. The significant difference in ratio values between two features is indicative that these differences will show up on a deeper level of BSD and features would not match at higher levels. However, we cannot say at what higher level it will actually happen. The probability to get match on the next BSD level is lower for shoulders with very different ratios than for shoulders with similar ratios. Our simulation experiments confirm this. It is also consistent with an expectation that the probability of a high level of match $(k>4)$ for images with significant noise and different resolution is low. Thus, we cannot expect many of these very good cases and having the majority of low-level matches the FA algorithm will significantly shorten computation time.

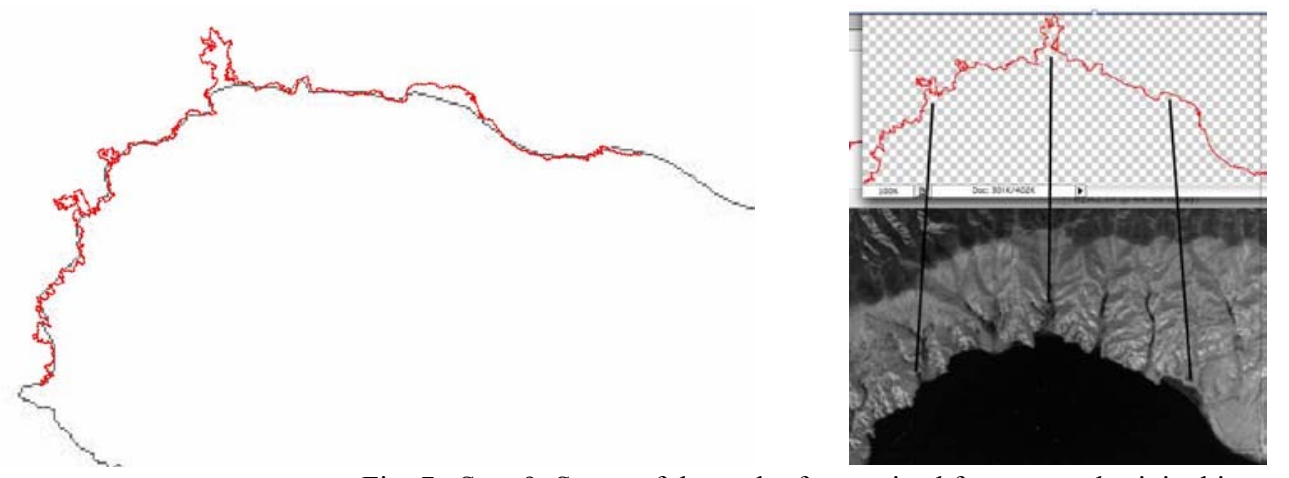

Fig. 7. Step 9: Successful match of vectorized features and original image.

\section{CUTTING UNITS FROM FEATURES}

The next step is to explore the situation when a feature match is not found. In this case, a search for matching subfeatures is initiated by cutting a predefined unit from the first superfeature and repeating, keeping other superfeatures unchanged. Currently we use a unit size between $1 / 200$ of the image largest measurement and half the size of a large feature. It is suggested to start from large units to save time.

If no match is found, the process is repeated by sequentially cutting units from all superfeatures until a match is found or the superfeatures are gone. Fig. 7 shows the final conflation result for the example discussed. This process forms steps 10 and 11 of the conflation algorithm:

Step 10. If Steps 7 and 8 fail cut a predefined unit from the first superfeature and repeat steps $4-9$ for this modified superfeature and unchanged other superfeatures.

Step 11. If match is not found repeat step 10 by sequentially cutting units from all superfeatures and until match will be found or superfeatures are completely cut down.

Optimizing unit size. The unit size $u$ used in steps 10 and 11 is the most critical parameter of the whole CM method. If the unit size is equal to the length $\mathrm{L}$ of the largest superfeature then steps 10 and 11 are empty. If the unit is a half of the $\mathrm{L}, u=\mathrm{L} / 2$ then at most step 11 will be repeated two times for each superfeature, that is total $n \times m$ times, where $n$ is the number of superfeatures in image A and $m$ is the number of superfeatures in image B. Our experiments show that 
three superfeatures per image were sufficient to find the match. Thus, $n \times m$ could be bounded by 9 times of running step 11. If each superfeature contains $r$ units then step 11 will be run $(r n) \times(r m)=r^{2} n m$ times, if there is no optimization of time. This is polynomial time complexity function. The combination of AA and SA algorithms provides such time optimization by cutting time of running step 11 itself by half.

\section{QUALITY OF CONFLATION}

Conflation performance is defined by the quality and time of conflation. We are measuring the quality of conflation by a certainty that features are matched correctly. The speed of conflation is measured by the time required to conflate images. The quality of conflation is a multidimensional characteristic. In this paper, we focus on how close structurally features are in the two images. For each pair of features, it is measured by the BSD level parameter $k$. We assume that $k \geq 5$ indicates a high quality of conflation match. The final match is done by checking that there are no other matching features nearby with the same or higher level of match. In this is the case, we continue BSD process with both AA and SA algorithms and all highly matched features to find a pair with the highest match.

Error estimates. We need to know how good the conflation result is. A standard approach is to measure the difference between control points after a matching transformation is completed. In the base case, the distance is 0 for all control points after transformation. Does it tell us how good the conflation is or how good is the match for other points? Say, we have 10 control points in an image of $1000 \times 1000$ pixels, that is 10 points out of $10^{6}$ points. This means that we evaluated the quality of conflation using $0.001 \%$ of the total number of points. The question is how we can evaluate the quality for the other $99.999 \%$ of the points without actually having $10^{6}$ control points. The classical smooth interpolation approach does not answer this question explicitly. The only statement that can be made is that smooth continuous interpolations such as linear, polynomial and splines transfer close points to close points. But how close is not clear. An expectation is that the error for other points should be similar to the errors computed for control points. If we have zero error for $0.001 \%$ of the total number of points, we can not claim that we will have zero error for the rest of the image too. Thus an innovative approach is needed to measure quality of conflations.

Matching non-control points. A transformation $\mathrm{T}$ based on control points will also transform all other image points, $T(x)=y$. But it is not done specifically for them. It is a byproduct of matching control points. Why should point $x$ be matched to point $y$ if $x$ is not a control point? There is a huge number of transformations $T$ ' that match control points, but differ from $T$ in other points. Transformation $T^{\prime}$ can convert point $x$ to points $y$ ' that can be close to $y$ but not identical to $y$. Our conflation method identifies matched non-control points in a more meaningful way using a combination of measures provided by AA and SA algorithms. This is important for enhancing a standard control point matching approach.

Virtual imagery expert. In addition, we are automatically capturing some human abilities to match images using context. In Fig. 3 we may assume that an expert knows that the left image is a low-resolution image and the right image is a high-resolution image. Such context knowledge permits the expert to match these features despite their apparent differences. The combined AA and SA algorithm can help to discover this from the image itself by analyzing shoulder relations .

\section{ROBUSTNESS OF ALGORITHMS FOR IMAGES WITH DIFFERENT RESOLUTION AND NOISE LEVEL}

One of the major conflation challenges is matching features from images with significantly different resolution. Feature extraction artifacts can also contribute to feature disparity. As we have seen in Fig. 3, such features have different levels of smoothness and detail. In Fig. 8 we show the behavior of the SA algorithm in the presence of random noise that simulates effect of difference in resolution. Binary sequential division (BSD) was applied two times and produced a polyline shown in Fig. 8. 
To build structural matrices we compute all $\mathrm{S}_{\mathrm{i}} / \mathrm{L}_{\mathrm{i}}$ ratios for the low resolution line (straight line) and the high resolution line with spikes. Here $\mathrm{L}_{\mathrm{i}}$ is the length of the $i$-th polyline segment along the curve and $\mathrm{S}_{\mathrm{i}}$ is the length of the straight line between end points of the $i$-th polyline segment. These ratios are: 1,1,1,1 for the straight line and, say, 0.4 , $0.45,0.48$, and 0.51 for the curve. Next, we check that all these ratios are close enough in each feature separately, $\left|\mathrm{S}_{\mathrm{i}}-\mathrm{S}_{\mathrm{i}}\right|<\varepsilon$ for $\varepsilon=0.15$. All pairs of ratios are in these limits in both features. Thus, the SA algorithm matches given features on this BSD level. Significant differences in ratio values between two features are indicative that on a deeper level of BSD these differences will show up and features will not match at higher levels.

Similarly, we can compute three sequential angles $\left(170^{\circ}, 165^{\circ}\right.$, and $\left.175^{\circ}\right)$ for the high resolution feature. These angles are all $180^{\circ}$ for the straight line. All angle differences are less than the $15^{\circ}$ limit for both images. Thus they are matched by the AA algorithm on this BSD level. But the AA algorithm does not give any clue that this match may deteriorate in the next levels, in contrast with the SA algorithm that provides such clues.

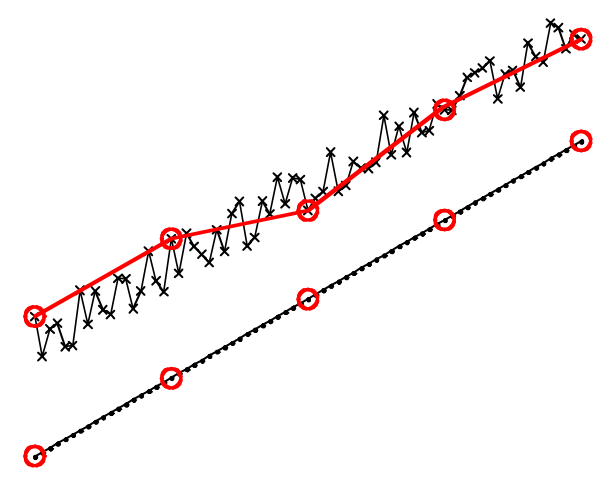

Fig. 8. This example indicates that the SA algorithm based on lengths is more robust for similar cases than the AA algorithms based on angles.

\section{GENERALIZATION: IMAGE STRUCTURAL SIMILARITY}

It is critical to measure the quality of the whole registration/conflation process. We described the issue of matching individual features from two images using the concept of structural equivalence of individual features. Now this concept is used as a base to define structural similarity of two images. No single numeric measure is sufficient to cover the complex variability of images and matching contexts/situations. Thus, we introduce a set of measures that is a more adequate measure of the quality of registration/conflation.

We start with the simple situation with one-to-one feature matches. Every feature in image 1 has a single match in image 2 and the same is true for the image 2 . Let $n=\left(n_{1}, n_{2}, \ldots, n_{m}\right)$ be a vector of structural similarities defined above for all $\mathrm{m}$ pairs of matched features. This vector provides a measure of the structural similarity of two images. We can compute $\max \left(n_{1}, n_{2}, \ldots, n_{m}\right), \min =\left(n_{1}, n_{2}, \ldots, n_{m}\right)$, average $\left(n_{1}, n_{2}, \ldots, n_{m}\right)$ and the variety of moments of the distribution of $\left(n_{1}, n_{2}, \ldots, n_{m}\right)$ and use them as similarity indicators if $\mathrm{m}$ is a large number. The difference $\max \left(n_{1}, n_{2}, \ldots, n_{m}\right)$ $\min \left(n_{1}, n_{2}, \ldots, n_{m}\right)$ can be used as an indicator of variability in structural similarity. These indicators can also serve as change indicators that are important for image change detection tasks. High values of elements of vector $n$ mean that there is no change in the image 1 during the period between taking image 1 and image 2 .

Now we can consider the situation with subsets of features. Every feature in image 1 has a single match in image 2, but some features in image 2 have no matched feature in image 1. This situation is possible when there is a new development in the area. Again $n=\left(n_{1}, n_{2}, \ldots, n_{m}\right)$ is a vector of structural similarities for all $m$ pairs of matched features, but these pairs do not contain all the features in image 2 . Similarly we can compute $\max \left(n_{1}, n_{2}, \ldots, n_{m}\right), \min =\left(n_{1}, n_{2}, \ldots, n_{m}\right)$, average $\left(n_{1}, n_{2}, \ldots, n_{m}\right)$ and other characteristics listed above. However, now even if we have high values of elements of vector $n$ we cannot say that there is no change during the period between taking image 1 and image 2 . Let $m_{l}$ and $m_{2}$ be 
the number of features in Image 1 and Image 2, respectively. Then there is a vector $n$ with $m_{l}$ elements. A new indicator, $m_{1} / m_{2}$ shows the ratio of common features in two images that can be added. Similarly, sets of measures for more complex situations can be introduced.

The structural similarity definitions can be augmented with definitions of geometric similarity, based on metric distances. We may need to transform one image to the other one before computing geometric similarity. The transformation can be based on features that have been matched.

Below we summarize a version of our shoulder-based method to measure a structural difference in curvilinear features in the following way:

Step 1: Compute $\mathrm{L}$, the length of the line from its start to the middle point $\mathrm{D}$ along the line.

Step 2: Compute the lengths of two "shoulders", $S_{1}$ and $S_{2}$, that is $S_{1}$ the length of the straight line [T,D] between the start point $\mathrm{T}$ and the middle point $\mathrm{D}$.

Step 3. Compute ratios $R_{1}=S_{1} / L_{1}$ and $R_{2}=S_{2} / L_{2}$, where $L_{1}=L_{2}$ is the half feature length computed along the feature. If a ratio $R_{1}$ is close to 1 then the first part of the feature is close to the straight line, similar with $R_{2}$. Find ratios that are in the threshold limit. .

Step 4. Repeat steps 1-3 for subfeatures [T, D] and [D,E] recurrently for their subfeatures until all ratios are equal to 1 (straight line).

Theorem. For any polyline with $n$ nodes, step 4 must be repeated no more than $n \lg n$ times to get all ratios equal to 1 .

Proof. This can be shown by considering an extreme case, where after the finding a middle point $\mathrm{D}$, only the single end points will be in the right "shoulder". This means that the $n-1$ nodes are in the part between the start node T and M including node $\mathrm{p}_{\mathrm{n}-1}$. We know that the polyline between node $\mathrm{p}_{\mathrm{n}-1}$ and the end node $\mathrm{E}=\mathrm{p}_{\mathrm{n}}$ is a straight line $\left[\mathrm{p}_{\mathrm{n}-1}, \mathrm{E}\right]$. Above we assumed that $\mathrm{M}$ is between them, thus $[\mathrm{D}, \mathrm{E}]$ is a straight line and a part of the longer straight line $\left[\mathrm{p}_{\mathrm{n}-1}, \mathrm{E}\right]$. That is the ratio $R_{2}$ is equal to 1 for $[D, E]$ and Step 4 took only one iteration for the subfeature between $D$ and $E$.

Now we assume that the same extreme case is true for the left subfeature from point $\mathrm{T}$ to point $\mathrm{D}$ and subsequently for all its subfeaures. That is we need to repeat step 4 only $n$ times for this extreme case. If our previous assumption is not true and we have more than one node in the subfeature between $\mathrm{D}$ and $\mathrm{E}$, we can repeat such binary search process at most for $\lg n$ times to come to the situation with a single straight line. Having total $n$ nodes it may take $n \lg n$ loops with Step 4 in the worst case.

\section{RELATED WORK ON CONFLATION MEASURES}

Characterizing and evaluating the quality of conflated data relative to a given problem is one of the most important open problems in geospatial data integration. A major part of this development is producing multidimensional measures of the correctness of conflation, since no single measure of quality of conflated data sets is appropriate for every conflation problem. Traditionally registration, co-registration, and conflation have been done with images of a similar type. Some processes have used every pixel or every vector in each image. For these processes, the feature space is essentially the image and the similarity metrics chosen to guide the process and the transformations allowed between the images have an important bearing on the outcome as does the presence of local minimums in the search space and the search strategy. Other conflations use feature spaces that have been derived from the images using a variety of techniques including the raw intensities, extractable edges, salient and statistical features, matches against models, and so on. There are numerous possible choices for similarity metrics used for both images and features including, crosscorrelation with and without pre-filtering, correlation coefficients, phase-correlations, sums of absolute differences of intensity, sums of absolute differences of contours, other contour/surface differences, number of sign changes in pointwise intensity differences, sums of squares of differences between nearest points, minimum changes in entropy, etc.

We focus on three methods that were developed for processes similar to ours. The first is a technique of matching planar polylines described by Cohen and Guibas ${ }^{9}$. We analyzed their task, called the polyline shape search problem or PSSP for short, to clarify the applicability of their approach to the algebraic structural conflation (ASC) task 
formalized here and to compare the differences in task formalizations and interpretations. Our conclusions are as follows:

(1) Both tasks PSSP and ASC assume that matching may require rotation, scaling and translation of polylines.

(2) The task of PSSP is to find a part of a polyline A that matches a polyline B. Polyline B can be a relatively small "template" polyline (e.g., corner-shape or step-shape). See Fig. 9.

(3) The task of ASC is to find the largest matched part C of two larger polylines A and B.

(4) PSSP uses two criteria for judging the quality of a match:

- the error of the match computed as a distance between polyline A and the matched part of polyline B after B is transformed by shifting, rotating and scaling.

- the length of the match is computed as a number of matched breakpoints on the polylines multiplied by $\alpha$ (stretching parameter). This parameter is used to transform $\mathrm{B}$ to $\mathrm{A}$.

Our ASC method is more general than the one developed by Cohen and Guibas because (1) two arbitrary polylines can be searched for similar portions in each and (2) there are no point limitations on the determination of distances. Another measure of feature shape similarity was defined by Cobb et $\mathrm{al}^{7}$. The idea of the method is illustrated in Fig. 10 (a) and (b).

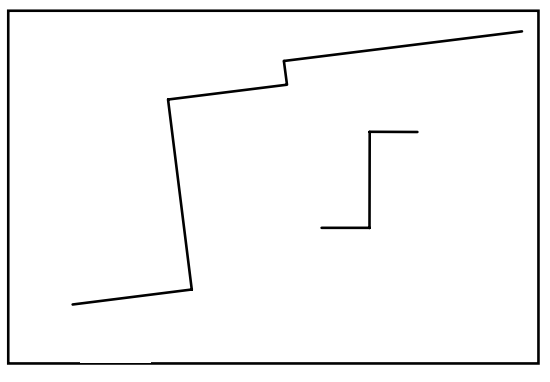

Fig. 9. Template examples PSSP.

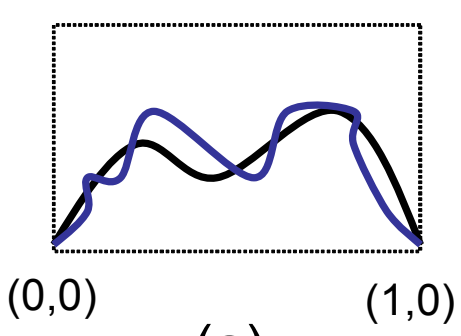

(a)

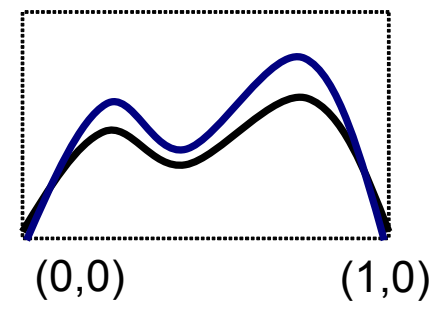

(b)

Fig. 10: Structural differences

The measure of Cobb et al. is based on the following steps:

1. Bring the features to a standard position with the start point $(\mathrm{x}, \mathrm{y})=(0,0)$ and the end point $(\mathrm{x}, \mathrm{y})=(1,0)$ by rotating and rescaling the features. In this way, feature 1 is presented as a function $P:[0,1] \rightarrow R^{2}$ and feature 2 is presented as a function $\mathrm{Q}:[0,1] \rightarrow \mathrm{R}$.

2. Merge nodes -- make the number of nodes and their locations on the $x$ coordinate equal in both features by mapping all nodes $\left(\mathrm{p}_{0}, \mathrm{p}_{1}, \ldots, \mathrm{p}_{\mathrm{n}}\right)$ from feature 1 onto the feature 2 , and by mapping all nodes $\left(\mathrm{q}_{0}, \mathrm{q}_{1}, \ldots, \mathrm{q}_{\mathrm{m}}\right)$ from feature 2 onto feature 1 . The merged number of nodes is $n+m$ if there are no overlapping nodes in features.

3. Compute the Frechet measure of distance $L_{2}$ between features as a square root of the integral of squared differences between features. Technically, this is done by using merged nodes.

$$
\mathrm{L}_{2}=\left(\int_{[0,1]}\|P(r)-Q(r)\|^{2} d r\right)^{1 / 2}=\left(\sum_{\mathrm{i}=1, \ldots, \mathrm{n}+\mathrm{m}} \int_{[\mathrm{Y}(\mathrm{i}-1), \mathrm{Y}(\mathrm{i})]}\|P(r)-Q(r)\|^{2} d r\right)^{1 / 2}
$$

4. Compute the normalized distance between features, $L_{2}\left(D_{1}+D_{2}\right)$, where $D_{1}$ and $D_{2}$ are lengths of the features in the standard position.

This measure is applicable for the following situations:

- Features can be arbitrarily rotated and have different sizes.

- Ratios of scales for $x$ and $y$ are similar for both features. Fig. 10 (a) shows this case and Fig. 11 (b) shows the significant difference in ratios in two images indicated by dotted rectangles. In Fig. 11 (a) Feature 1 is taken from the image where the ratio of width and height of the feature bounding box is 0.5 and feature 2 is taken from the image 2 where the ratio is 0.6 .

- Some structural differences are not important. Fig. 10 (a) shows structural differences that are not captured by the measure; the higher lines in both Fig. 10 (a) and Fig. 10 (b) may give the same similarity with the lower line. 
- Each feature is in the bounding box defined by the start and end points, see Fig. 11 (c). Note this method is not applicable for features shown in Fig. 11 (d).

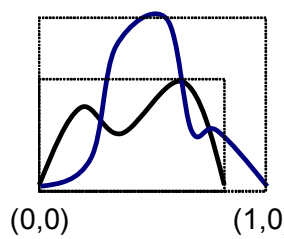

(a)

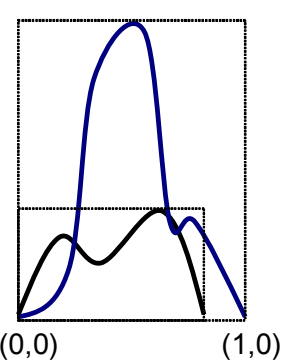

(b)

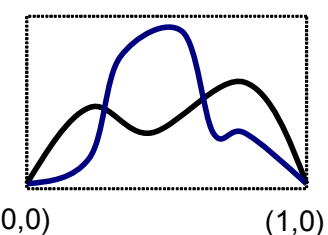

(c)

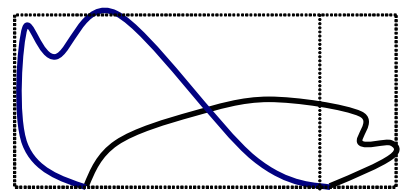

$(0,0)$
$(1,0)$

(d)

Fig. 11. Examples of scale ratios and Bounding boxes for standardized features.

Our ASC method described earlier is applicable for both conflation cases presented in Fig. 11. It captures the structural differences between two lines shown in Fig. 10(b) using angles.

While the method of Cobb is satisfactory for similar features with similar lengths, it lacks the ability to determine partial matches of polylines. By using the Frechet measure of distance $L_{2}$ between features it cannot accurately compare structures that are very different and will not work at all with others such as illustrated in Fig. 11 (d).

Carswell et al. ${ }^{9}$ have developed a composite similarity metric to aid in the location of "image-objects" in images. They combine weighted topology, orientation, and relative-distance similarity measures for the image components $Q$ and compare it with an image scene $I$. For instance for finding a house with an outdoor swimming pool across a road from a barn and silo in the image, the "image-object" would consist of these five elements with appropriate topology, orientations, and distances defined. Their similarity measure provides a percentage match between the sum of these features $Q$ and similar ones in the image $I$.

The individual similarity measure weights can be varied to emphasize the relative importance of different image properties. For the example above, one could weight the topological relationships strongly, the relative-distance measure moderately and the orientation similarity metric the least.

This approach should give similar results to one that applies algebraic invariants by combining individual features into composite features and then proceeding with the conflation/co-registration process in a normal manner with loosened distance matching constraints.

\section{SUMMARY}

In a traditional control point approach to imagery conflation and registration, the matching of non-control points is a byproduct of matching control points. If $x$ is not a control point it is not clear why it should be matched to point $y$ by a specific transformation $T$. There is a myriad of transformations $T$, that match control points, but differ from $T$ in transforming other points. This paper shows how matching features and their non-control points can be done in a more meaningful way using the ideas of abstract algebraic structures and relations.

\section{ACKNOWLEDGEMENTS}

The authors gratefully acknowledge that this research has been supported by National Geospatial-Intelligence Agency through its Academic Research Program Grants NMA401-02-1-2006 and NMA401-02-1-2007. 


\section{REFERENCES}

1. Aronov, B., R. Seidel and D. Souvaine. On compatible triangulations of simple polygons. Comput. Geom. Theory Appl. 3, 27-35, 1993.

2. Bartl, R., and W. Schneider. Satellite image registration based on the geometrical arrangement of objects. Proc. SPIE, v. 2579, p. $32-40,11 / 1995$.

3. Bern M. et al. Emerging challenges in computational topology. citeseer.nj.nec.com/bern99emerging.html, 1999.

4. Boyce, R., Chase, R., Donovan, T., and Frank, E. MBP Conflate, Computer Science 481, technical report, Central Washington University, 2003.

5. Cohen, J., Varshney, A., Manocha, D., Turk, G., Weber, H., Agarwal, P., Brooks Jr., F., and Wright, W. Simplification envelopes. In Rushmeier, H., Ed., Proc. SIGGRAPH '96, 119-128. Addison Wesley, 1996.

6. Cohen, S. and L. Guibas, Partial Matching of Planar Polylines under Similarity Transformations. Proceedings of the Eighth Annual ACM-SIAM Symposium on Discrete Algorithms, 777-786, January 1997.

7. Cobb, M., Chung, M. J., Foley, H., Petrie, F., and Shaw, K. A rule-based approach for the conflation of attributed vector data, Geoinformatica 2:1, 7-35, 1998.

8. Chase R., and Kovalerchuk B., Computationally efficient algorithm for structural feature matching, In: Proceedings of International Conference on Imaging Science, Systems, and Technology (CISST'2003), Las Vegas, p. 253-259, 2003.

9. Carswell, J. D., Wilson, D. C., and Bertolotto, M. Digital Image Similarity for Geo-spatial Knowledge Management, in Advances in Case-Based Reasoning, Springer-Verlag, Berlin, 2002.

10. Dare, P. M. and Dowman, I. J. Automatic registration of SAR and SPOT images based on multiple feature extraction and matching. In: Proceedings of IEEE International Geoscience and Remote Sensing Symposium, Honolulu, Hawaii, 2000.

11. Dare, P. M. Automatic registration of multi-source remotely sensed images and other non-image spatial information products, University of Melbourne, Australia, www.sli.unimelb.edu.au/pdare/arspc dare.pdf, 2000.

12. Dey T., Guha, S. Computing homology groups of simplicial complexes. JACM, 45(2):266-287, March 1998.

13 Dey, T., H. Edelsbrunner, and S. Guha. Computational Topology. In Advances in Discrete and Computational Geometry (Contemporary mathematics 223), ed. B. Chazelle, J. E. Goodman, and R. Pollack, American Mathematical Society, 109-143, 1999. http://citeseer.nj.nec.com/dey99computational.html

14. Eppstein D., Geometry in action: Cartography and Geographic Information Systems http://www.ics.uci.edu/ eppstein/gina/carto.html

15. Gillman D., Triangulation for rubber-sheeting, Auto Cartography 7, 191-197, 1985.

16. Gottesfeld Brown, L. A survey of image registration techniques. ACM Computing Surveys, 24(4):325--376, December 1992.

17. Gupta, H., and Wenger, R. Constructing pairwise disjoint paths with few links. Technical Report OSU-CISRC-2/97-TR16, The Ohio State University, Columbus, Ohio, 1997. http://citeseer.nj.nec.com/gupta97constructing.html

18. Hirose, M., Furuhashi, H., Kitamura, N., and Araki, K. Method for automatic registration using real-time multiview range images. Proc. SPIE Vol. 4572, p. 174-182, 10/2001.

19.Kovalerchuk B., Sumner W., Algebraic relational approach to conflating images, In: Algorithms and technologies for multispectral, hyperspectral and ultraspectral imagery IX. Vol. 5093, International SPIE Military and Aerospace symposium, AEROSENSE, Orlando, FL., Apr.21-25, pp. 621-630, 2003.

20. Kovalerchuk, B. and Schwing, J. Algebraic relational approach for geospatial feature correlation, In: Proceedings of International Conference on Imaging Science, Systems, and Technology (CISST'2002, June 24-27, 2002), Las Vegas, pp. 115-121, 2002, http://www.cwu.edu/ borisk/pub/CWUMethod.pdf

21. Mal'cev A.I. Algebraic Systems, Springer-Verlag, New York, 1973.

22. Pinz, A., Prantl, M., and Ganster, H. A robust affine matching algorithm using an exponentially decreasing distance function. J.UCS - Journal of Universal Computer Science, Springer, 1(8), 1995.

23. Stiles, D. NIMA Teams with Military Services to Define Mission-Specific Feature Data, In: Pathfinder, Bethesda, p. 4, May/June 2003.

24. Wang, J., Chun, J., and Park, Y.W. GIS-assisted image registration for an onboard IRST of a land vehicle. Proc. SPIE Vol. 4370, p. 42-49, 2001.

25. Ziou, D., and Tabbone, S. Edge Detection Techniques- An Overview, technical report, No. 195, Dept Math \& Informatique. Universit de Sherbrooke, 1997. 